\title{
Enseñanza virtual en educación física en primaria en México y la pandemia por COVID-19
}

\author{
Virtual education in physical education for elementary school in Mexico \\ and the COVID-19 pandemic
}

\section{* Javier Arturo Hall López \& ** Paulina Yesica Ochoa-Martínez}

Hall, J. A., \& Ochoa-Martínez, P. Y. (2020). Enseñanza virtual en educación física en primaria en México y la pandemia por COVID-19. Revista Ciencias de la Actividad Física UCM, № 21(2), julio-diciembre, 1-7. DOI: http://doi.org/10.29035/rcaf.21.2.4

\section{RESUMEN}

La revisión teórica aborda la educación física virtual establecida en México a partir del confinamiento social por COVID-19, basada en el problema, ya existente en México, de sedentarismo y obesidad infantil en niños de educación primaria, en quienes, derivado del confinamiento social, se estima un aumento de masa corporal, lo que conlleva mayores riesgos de salud. Al analizar la bibliografía reciente, que relaciona al COVID-19 con la educación física y la actividad física, se identifica la oportunidad del profesorado de educación física, de conducir esquemas virtuales en los contenidos del programa educativo para, de esta manera, coadyuvar en la disminución del sedentarismo en estudiantes de primaria, teniendo como prioridad la alfabetización física.

Palabras clave: educación física, educación primaria, educación virtual, COVID-19.

\section{ABSTRACT}

The theoretical review addresses the virtual physical education established in Mexico based on social confinement by COVID-19, based on the problem, already existing in Mexico, of sedentary lifestyle and childhood obesity in primary school children, in whom, derived from social confinement, an increase in body mass is estimated, which carries greater health risks. When analyzing the recent bibliography, which relates COVID-19 with physical education and physical activity, the opportunity for physical education teachers to conduct virtual schemes in the contents of the educational program is identified, in this way, to contribute to the decrease in sedentary lifestyle in primary school students, prioritizing physical literacy.

Key words: Physical education, Elementary Education, Virtual Education, COVID-19.

\footnotetext{
* Universidad Autónoma de Baja California, Facultad de Deportes, Mexicali, México. javierhall@uabc.edu.mx
}

** Universidad Autónoma de Baja California, Facultad de Deportes, Mexicali, México. pochoa@uabc.edu.mx 
Hall, J. A., \& Ochoa-Martínez, P. Y. (2020). Enseñanza virtual en educación física en primaria en México y la pandemia por COVID-19. Revista Ciencias de la Actividad Física UCM, № 21(2), julio-diciembre, 1-7. DOI: http://doi.org/10.29035/rcaf.21.2.4

\section{Introducción}

La pandemia por COVID-19 representa un reto de salud pública, que requiere en todos los sectores sociales un esfuerzo urgente y colaborativo para disminuir el riesgo de salud y la pérdida de vidas de la población (Barrientos, Alpuche, Lazcano, Pérez \& Rivera, 2020). Derivado del COVID-19, en diversos sectores de la población se estableció el confinamiento, que incluyó la suspensión de actividades escolares con seguimiento virtual por parte del profesorado y la tutela de la familia acompañando al estudiantado, para dar continuidad a los contenidos en los planes de estudio (Cóndor, 2020).

La emergencia sanitaria llegó de manera súbita y el profesorado, bajo esta circunstancia, exigidos por la pandemia de manera inesperada, ajustaron y adecuaron sus acciones pedagógicas cotidianas, previamente planeadas para innovar en la atención al estudiantado de manera virtual, preparándose y obteniendo experiencia en el uso de tecnologías simultáneamente (Lloyd, 2020). En ese sentido, la clase de educación física en educación básica formó parte de estas adecuaciones curriculares (Gómez, 2020); cuya manera tradicional de atención educativa al estudiantado es en un espacio extenso para el movimiento acorde a los objetivos de la clase, pero debido a la pandemia por COVID-19, el confinamiento social ha limitado la práctica de actividad física deportiva o ejercicio (Blocken, Malizia, van Druenen \& Marchal, 2020), por lo que se prevé que durante la pandemia por COVID-19 exista un incremento de enfermedades cardiovasculares asociadas al sedentarismo (Lippi, Henry \& Sanchis, 2020). En México, lo anterior incrementa el problema de salud asociado a enfermedades crónicas no trasmisibles por las altas prevalencias de sedentarismo, sobrepeso y obesidad, disminuyendo la calidad de vida de los niños y niñas de educación primaria (Aguilar, Rodríguez, Menor, Guisado, León \& Sánchez, 2019), por lo que se identifica, desde el ámbito educativo, al profesor de educación física para coadyuvar en la solución de la problemática al ser considerado un profesional que, en el contexto educativo y de salud pública, está capacitado para orientar la disminución del sedentarismo (Webster, Webster, Russ, Molina, Lee \& Cribbs, 2015).

Por lo anterior, en la presente revisión teórica se presenta de manera analítica a partir de recientes referencias y antecedentes bibliográficos sobre la actividad física y la educación física durante la pandemia por COVID-19, una reflexión crítica del desarrollo y procedimientos de adecuaciones curriculares en estudiantes de educación primaria por el profesorado de educación física, mediante el uso de educación virtual, en el contexto educativo Mexicano.

\section{Antecedentes}

Como antecedentes se presentan los resultados de la Encuesta Nacional de Salud y Nutrición (ENSANUT), cuyas prevalencias combinadas de sobrepeso y obesidad están reportadas en tres de cada diez niños y niñas de edad escolar (cinco a once años de edad), dichos valores son de los altos a nivel mundial (Pérez \& Cruz, 2019), y tomando en consideración lo establecido por Rundle, Park, Herbstman, Kinsey \& Wang, (2020), que estima que después de la pandemia por COVID-19, se espera que los estudiantes de edad escolar presenten un aumento de masa corporal derivado del sedentarismo por el confinamiento y la limitación de la actividad física.

El problema se hace aún más grave para edades infantiles, por lo que se recomienda la práctica de actividad física moderada a vigorosa en el periodo de aislamiento social (Cossio, 2020). Misma que en niños y niñas la Organización Mundial de la Salud (OMS, 2010), establece como adecuada para la salud, con una dedicación mínima de 60 minutos diarios de actividad física con intensidad moderada a vigorosa; la actividad física diaria debería ser, en su mayor parte aeróbica y convendría incorporar, como mínimo tres veces por semana, actividades vigorosas que refuercen, en particular, los músculos y huesos. La actividad física con intensidad moderada a vigorosa se ha recomendado como la más efectiva durante el confinamiento por el COVID-19 (Dixit, 2020), 
Hall, J. A., \& Ochoa-Martínez, P. Y. (2020). Enseñanza virtual en educación física en primaria en México y la pandemia por COVID-19. Revista Ciencias de la Actividad Física UCM, № 21(2), julio-diciembre, 1-7. DOI: http://doi.org/10.29035/rcaf.21.2.4

de igual manera, el Colegio Americano de Medicina del Deporte (ACSM) recomienda la actividad física para el adecuado desarrollo inmunitario en niños (Radom, 2020). Como ejemplo para esta intervención, existen abordajes realizados en el ámbito de la educación física en educación primaria, que utilizaron la tecnología y ambientes virtuales, mejorando hábitos de salud y aumentando la actividad física en el estudiantado (Bezkopylnyi, Bazylchuk, Sushchenko, Bazylchuk, Dutchak \& Ostapenko, 2020, Oleniev, 2020).

\section{Desarrollo}

Bajo la crisis de salud por el COVID-19, en el sistema educativo mexicano, la clase de educación física, para el seguimiento y continuidad de los contenidos, presenta las mismas condiciones de educación virtual que las demás asignaturas. Se puede sacar ventaja de dos condiciones del perfil de egreso para educación primaria, que son: la atención al cuerpo y la salud, y las habilidades digitales, para trabajar en el componente curricular de educación física, como área de desarrollo personal social, cuyo objetivo en el programa es la formación integral de niñas, niños y adolescentes al desarrollar su motricidad e integrar su corporeidad (SEP, 2017).

Con la pandemia por COVID-19, existe un reto pedagógico del profesorado de educación física en primaria utilizando un contexto diferente al tradicional, fuera de la escuela, lo cual establece un esquema virtual, con diferentes posibilidades para que el estudiantado sea guiado en el desarrollo de su motricidad, complicando la posibilidad de retroalimentar, corregir, supervisar el movimiento y evaluar el desarrollo y evolución del aprendizaje (Gambau i Pinasa, 2020), por lo que la función del profesorado de educación física en los estudiantes de educación primaria es la alfabetización física como prestación estructurada, que se alcanza cuando los alumnos encuentran una gama de oportunidades apropiadas en cada etapa y edad (Frizzo \& Silva, 2019).

\section{Propuesta}

La clase de educación física es conducida en un amplio espacio para el movimiento de los alumnos, utilizando materiales e instrumentos, acorde a los objetivos de la sesión, donde el estudiantado se acompaña bajo la guía del profesorado que controla los factores para, de manera intencionada, llegar al objetivo planteado, con tareas motrices y estrategias didácticas acorde a la edad (Hall, Ochoa, Zuñiga, Macías \& Sáenz, 2017, Hall, Ochoa, González \& Fernández, 2019).

Con esta intención, durante el periodo de aislamiento social por la pandemia por COVID-19, el profesorado se ha propuesto la regulación gráfica de la intensidad de la actividad física en niños, niñas y adolescentes de edad escolar (Rodríguez, 2020), para mantener la condición física (Mera, Tabares, Montoya, Muñoz \& Monsalve, 2020). En España, el Consejo General de la Educación Física y Deportiva de España (Consejo COLEF), estableció directrices de actuación para el regreso a la normalidad de atención al estudiantado en clase de educación física (Consejo COLEF, 2020), de igual manera, en China, país de origen del COVID-19, se cuenta con especificaciones de intervención en el ámbito de la educación física, para que el profesorado implemente estrategias didácticas, que minimicen la propagación de la infección (Chen, Mao, Nassis, Harmer, Ainsworth \& Li, 2020)

Uno de los retos en el contexto mexicano, es la alfabetización en salud pública ante la emergencia de la pandemia por COVID-19 (Lazcano \& Alpuche, 2020) que debe ir a la par con la alfabetización digital, la cual no está presente en la totalidad del profesorado para usar de manera adecuada las tecnologías de la información y comunicación y guiar efectivamente al estudiantado (Cabezas \& Casillas, 2017). 
Hall, J. A., \& Ochoa-Martínez, P. Y. (2020). Enseñanza virtual en educación física en primaria en México y la pandemia por COVID-19. Revista Ciencias de la Actividad Física UCM, № 21(2), julio-diciembre, 1-7. DOI: http://doi.org/10.29035/rcaf.21.2.4

\section{Conclusiones}

Los profesionales de educación física y del deporte tienen una gran oportunidad para brindar una atención de calidad (mejorar su imagen ante la sociedad), y al mismo tiempo estar preparados ante los retos y desafíos por posibles rebrotes de COVID-19, que alarguen el confinamiento social y limiten la educación presencial (Sánchez, Arce \& Rodríguez, 2020). El uso de la educación virtual, Appsy redes sociales se convierten en una herramienta de gran utilidad para su aplicación en las ciencias de la actividad física (Melnyk, 2017, Ashanin, Filenko, Pasko, Poltorastskaya \& Tserkovna, 2017), así como su adaptación a la práctica docente en educación física en el nivel de primaria, intentando mantener al estudiantado con niveles bajos de sedentarismo y, por el contrario, incrementar los niveles de actividad física durante la pandemia por COVID-19 (Chen et al., 2020).

\section{REFERENCIAS BIBLIOGRÁFICAS}

Aguilar-Cordero, M., Rodríguez-Blanque, R., MenorRodríguez, M., Guisado-Barrilao, R., LeónRíos, X., \& Sánchez-López, A. (2019). Influencia de la actividad física sobre la calidad de vida de los niños con sobrepeso u obesidad. Salud Pública de México, 67(4), 550-551. DOI: https://doi.org/10.21149/10013

Ashanin, V., Filenko, L., Pasko V., Poltorastskaya, A., \& Tserkovna, O. (2017). Informatization on the physical culture of students using the «physical education» computer program. Journal of Physical Education and Sport, 17(3), 1970-1976. DOI: 10.7752/jpes.2017.03195

Barrientos-Gutiérrez, T., Alpuche-Aranda, C., Lazcano-Ponce, E., Pérez-Ferrer, C., \& Rivera-Dommarco, J. (2020). La salud pública en la primera ola: una agenda para la cooperación ante Covid-19. Salud Pública de México, 62(5), 598-606. DOI: https://doi.org/10.21149/11606
Bezkopylnyi, O., Bazylchuk, O., Sushchenko, L., Bazylchuk, V., Dutchak, Y., \& Ostapenko, H. (2020). Peculiarities of application of interactive educational technologies in training of future teachers of physical culture to work with health protection in secondary school. Journal of Physical Education and Sport, 20(S1), 291-297. Recuperado de https://efsupit.ro/images/stories/februarie2020/Art\%2040.pdf

Blocken, B., Malizia, F., van Druenen, T., \& Marchal, T. (2020). Towards aerodynamically equivalent COVID-19 1.5 m social distancing for walking and running. Urban Physics, Wind Engineering \& Sports Aerodynamics. Recuperado de http://www.urbanphysics.net/Social\%20Dis tancing\%20v20_White_Paper.pdf

Cabezas González, M., \& Casillas Martín, S. (2017). ¿Son los futuros educadores sociales residentes digitales? Revista electrónica de investigación educativa, 19(4), 61-72. DOI: https://doi.org/10.24320/redie.2017.19.4.1369

Chen, P., Mao, L., Nassis, G. P., Harmer, P., Ainsworth, B. E., \& Li, F. (2020). Returning Chinese school-aged children and adolescents to physical activity in the wake of COVID-19: Actions and precautions. Journal of Sport and Health Science, 9(4), 322-324. DOI: https://doi.org/10.1016/j.jshs.2020.04.003

Consejo COLEF (2020). Recomendaciones docentes para una educación física escolar segura y responsable ante la "nueva normalidad". Minimización de riesgos de contagio de la COVID-19 en las clases de EF para el curso 2020-2021. Revista Española de Educación Física y Deportes, 429, 81-93. Recuperado de https://www.reefd.es/index.php/reefd/article/view/902/748

Cóndor-Herrera, O. (2020). Educar en tiempos de COVID-19. CienciAméRica, 9(2), 31-37. DOI: http://dx.doi.org/10.33210/ca.v9i2.281 
Hall, J. A., \& Ochoa-Martínez, P. Y. (2020). Enseñanza virtual en educación física en primaria en México y la pandemia por COVID-19. Revista Ciencias de la Actividad Física UCM, N²1(2), julio-diciembre, 1-7. DOI: http://doi.org/10.29035/rcaf.21.2.4

Cossio-Bolaños, M. (2020). Actividad física en tiempos de cuarentena por el COVID-19 en niños y adolescentes. Revista peruana de ciencias de la actividad física y del deporte, 7(2), 913-914. Recuperado de https://www.rpcafd.com/index.php/rpcafd/ article/view/92

Dixit, S. (2020). Can moderate intensity aerobic exercise be an effective and valuable therapy in preventing and controlling the pandemic of COVID-19? Medical Hypotheses, 143, $109854 . \quad$ DOI: https://doi.org/10.1016/j.mehy.2020.109854

Frizzo, G., \& Silva Souza, M. (2019). Educação física nas diretrizes da unesco: o paradigma da aptidão física e da saúde na formação do capital humano. Movimento. Revista Educação Física da UFRGS, 25, e25022. DOI: https://doi.org/10.22456/19828918.76037

Gambau i Pinasa, V. (2020). COVID-19: La crisis ha afectado a todos. Revista Española de Educación Física y Deportes, (429), 15-18. Recuperado de https://www.consejocolef.es/post/covid19-vgambau3

Gómez Calvo, J. L. (2020). ...Y de pronto el covid-19 nos hizo despertar. Revista Española de Educación Física y Deportes, 429, 95-100. Recuperado

de: https://www.agesport.org/y-de-pronto-elcovid-19-nos-hizo-despertar-por-jose-luisgomez-calvo/

Hall-López, J. A., Ochoa-Martínez, P. Y., Zuñiga Burruel, R., Macías Castro, R., \& Sáenz-López Buñuel, P. (2017). Moderate-to-vigorous physical activity during recess and physical education among Mexican elementary school students (Actividad física moderada a vigorosa durante el recreo y clase de educación física en niños mexicanos de escuela primaria). Retos, (31), 137-139. Recuperado

de https://recyt.fecyt.es/index.php/retos/article /view/49640

Hall-López, J. A., Ochoa-Martínez, P. Y., González, C., \& Fernández Ozcorta, E. J. (2019). Clases de Educación Físicas activas mediante evaluación SOFIT. E-motion. Revista de Educación, Motricidad e Investigación, (13), 31-42. Recuperado de http://rabida.uhu.es/dspace/handle/10272/1 7268

Lazcano-Ponce, E., \& Alpuche-Aranda, C. (2020). Alfabetización en salud pública ante la emergencia de la pandemia por Covid-19. Salud Pública de México, 62(3), 331-340. DOl: https://doi.org/10.21149/11408

Lippi, G., Henry, B. M., \& Sanchis-Gomar, F. (2020). Physical inactivity and cardiovascular disease at the time of coronavirus disease 2019 (COVID-19). European Journal of Preventive Cardiology 27(9), 906-908. DOI: https://doi.org/10.1177/2047487320916823

Lloyd, M. (2020). Desigualdades educativas y la brecha digital en tiempos de COVID-19. En H. Casanova Cardiel (Coord.), Educación y pandemia: una visión académica (pp. 115121). Ciudad de México: Universidad Nacional Autónoma de México, Instituto de Investigaciones sobre la Universidad y la Educación.

Mera, A., Tabares-Gonzalez, E., Montoya-Gonzalez, S., Muñoz-Rodriguez, D., \& Monsalve Vélez, F. (2020). Recomendaciones prácticas para evitar el desacondicionamiento físico durante el confinamiento por pandemia asociada a COVID-19. Universidad y Salud, 22(2), 166-177. DOl: https://doi.org/10.22267/rus.202202.188

Melnyk, Y. (2017). Monitoring of health culture formation in schoolchildren. Journal of Physical Education and Sport, 17(S4), 2073 2079.

Recuperado 
Hall, J. A., \& Ochoa-Martínez, P. Y. (2020). Enseñanza virtual en educación física en primaria en México y la pandemia por COVID-19. Revista Ciencias de la Actividad Física UCM, № 21(2), julio-diciembre, 1-7. DOI: http://doi.org/10.29035/rcaf.21.2.4

http://efsupit.ro/images/stories/30sept/Art \%20210.pdf

Oleniev, D. (2020). Research of health-preserving technologies in the system of physical education of students. Scientific Journal of National Pedagogical Dragomanov University, 2(122), 130-134. DOl: https://doi.org/10.31392/NPUnc.series15.2020.2(122).26

Organización Mundial de la Salud (OMS). (2010) Recomendaciones mundiales sobre actividad física para la salud. Recuperado de

https://apps.who.int/iris/bitstream/handle/1 0665/44441/9789243599977_spa.pdf

Pérez-Herrera, A., \& Cruz-López, M. (2019). Situación actual de la obesidad infantil en México. Nutrición Hospitalaria, 36(2), 463-469. Recuperado de http://scielo.isciii.es/scielo.php?script=sci_arttext\&pid=SO 212-16112019000200463

Radom-Aizik, S. (2020). COVID-19, Ejercicio, Niños y su Sistema Inmunitario en Desarrollo. Recuperado de https://www.exerciseismedicine.org/support_page.php/stories/?b=902

Rodríguez-Núñez, I. (2020). Prescribiendo ejercicio físico en períodos de cuarentena por COVID-19: ¿Es útil la autorregulación perceptual en niños? Revista Chilena de Pediatría, 97(2), 304-305. DOl: http://dx.doi.org/10.32641/rchped.v91i2.2208

Rundle, A. G., Park, Y., Herbstman, J., Kinsey, E. W., \& Wang, Y. C. (2020). COVID-19-Related School Closings and Risk of Weight Gain Among Children. Obesity, 28(6), 1008-1009. DOI: https://doi.org/10.1002/oby.22813

Sánchez-Duque, J. A., Arce-Villalobos, L. R., \& Rodríguez-Morales, A. J. (2020). Enfermedad por coronavirus 2019 (COVID19) en América Latina: papel de la atención primaria en la preparación y respuesta [Coronavirus disease 2019 (COVID-19) in
Latin America: Role of primary care in preparedness and response]. Atención primaria, 52(6), 369-372. DOl: https://doi.org/10.1016/j.aprim.2020.04.001

Secretaría de Educación Pública (SEP). (2017). Aprendizajes Clave para la Educación Integral. Educación Física. Educación Básica. Plan y Programas de Estudio y sugerencias de evaluación. México: Secretaría de Educación Pública. Recuperado de http://www.sep.gob.mx/work/models/sept/ Resource/10933/1/images/Aprendizajes_cla ve_para_la_educacion_integral.pdf

Webster, C. A., Webster, L., Russ, L., Molina, S., Lee, H., \& Cribbs, J. (2015). A systematic review of public health-aligned recommendations for preparing physical education teacher candidates. Research quarterly for exercise and sport, 86(1), 30-39. DOI: https://doi.org/10.1080/02701367.2014.98093 9 
Hall, J. A., \& Ochoa-Martínez, P. Y. (2020). Enseñanza virtual en educación física en primaria en México y la pandemia por COVID-19. Revista Ciencias de la Actividad Física UCM, N²1(2), julio-diciembre, 1-7. DOI: http://doi.org/10.29035/rcaf.21.2.4

\section{Dirección para correspondencia}

Dr. Paulina Yesica Ochoa-Martínez

Universidad Autónoma de Baja California, Facultad de Deportes, México.

Mexicali, México.

Dirección postal: Av. Rio Mocorito y Monclova Colonia Ex-ejido Coahuila Campus Mexicali II. Mexicali Baja California México.

ORCID y/o Reserach ID: https://orcid.org/0000-0001-8107-4906,

Contacto:

pochoa@uabc.edu.mx

Recibido: 10-07-2020

Aceptado: 28-08-2020

\section{(c) (i) (?)}

Esta obra está bajo una Licencia de Creative Commons Reconocimiento-NoComercialCompartirlgual 4.0 Internacional. 